\title{
Collaborative multidisciplinary design optimization: A framework applied on aircraft conceptual system design
}

\author{
Edris Safavi, Mehdi Tarkian, Hampus Gavel and Johan Ölvander
}

\section{Linköping University Post Print}

\section{Tweet}

N.B.: When citing this work, cite the original article.

Original Publication:

Edris Safavi, Mehdi Tarkian, Hampus Gavel and Johan Ölvander, Collaborative multidisciplinary design optimization: A framework applied on aircraft conceptual system design, 2015, Concurrent Engineering - Research and Applications, (23), 3, 236-249.

http://dx.doi.org/10.1177/1063293X15587020

Copyright: SAGE Publications (UK and US)

http://www.uk.sagepub.com/home.nav

Postprint available at: Linköping University Electronic Press

http://urn.kb.se/resolve?urn=urn:nbn:se:liu:diva-122539 


\title{
Collaborative Multidisciplinary Design Optimization - A Framework Applied On Aircraft Conceptual System Design
}

\author{
Edris Safavi \\ PhD Candidate, \\ Division of Machine Design, \\ Dept. of Management and \\ Engineering(IEI), \\ Linköping University, \\ Linköping SE-581 83 Sweden
}

\author{
Mehdi Tarkian \\ Assistant Professor, \\ Division of Machine Design, \\ Dept. of Management and \\ Engineering(IEI) \\ Linköping University, \\ Linköping SE-581 83 Sweden
}

\author{
Hampus Gavel \\ Section Manager, \\ Dept. of Modeling and \\ Simulation of Vehicle \\ System, \\ SAAB Aeronautics, \\ Linkoping, SE-581 88, \\ Sweden
}

\author{
Johan Ölvander \\ Professor, Division of Machine \\ Design, Dept. of Management \\ and Engineering(IEI) \\ Linköping University, \\ Linköping SE-581 83 Sweden
}

\begin{abstract}
In a product development process, it is crucial to understand and evaluate multiple and synergic aspects of systems such as performance, cost, reliability and safety. These aspects are mainly considered during later stages of the design process. However, in order to improve the foundations for decision-making, this paper presents methods that are intended to increase the engineering knowledge in the early design phases. In complex products, different systems from a multitude of engineering disciplines have to work tightly together. Collaborative design is described as a process where a product is designed through the collective and joint efforts of domain experts. A Collaborative Multidisciplinary Design Optimization (CMDO) process is therefore proposed in the conceptual design phase in order to increase the likelihood of more accurate decisions being taken early on. The performance of the presented framework is demonstrated in an industrial application to design aircraft systems in the conceptual phase.
\end{abstract}

Keywords: Conceptual Design, Collaborative Design, Aircraft system Design, Multidisciplinary Design optimization

\section{Introduction}

The conceptual design phase is one of the earlier phases of engineering design, where one or several design concepts are selected and optimized with respect to a set of initial requirements (Brandt et al. 1997). Hence, one of the main goals of a conceptual design study is to explore many feasible solutions and select a few of them for further analysis in later design phases (Ulrich et al., 2000), (Ullman D., 1992), (Pahl et al., 2007). Information gained during the conceptual phase creates a product knowledge foundation: gathering more information in early design stages is thus an advantage since it will promote better decision-making. This is especially true where complex and unconventional products with limited prior information are concerned (Wodehouse et al., 2010).

A complex product can be considered to be a single complex system with many subsystems working together. The working principles behind each subsystem may involve a multitude of engineering disciplines. Complex products are therefore multidisciplinary in their nature. Designing complex products involves the joint efforts of domain experts in various disciplines, each dependent on the work of others. The degree of complexity of a system depends on the number of components in the various domains used 
to create the system. The behavior of each component is defined by a set of design parameters and the relationships between them (Kroo et al., 1997).

Typically, complex products contain large numbers of components and accordingly large numbers of design parameters that are strongly coupled. The product architecture is thus defined as a linking together of various systems including different components which can affect many aspects of a complex product and its design quality such as functional performance, required design effort, production cost and ultimately customer satisfaction. Although system engineering or architecting is an activity classified as heuristics engineering, in the sense that they are often carried out by experienced systems engineers. However, considering all design parameters to obtain an optimized product seems to be hard and unfeasible even for domain experts (Ko, 2013). Multidisciplinary design optimization (MDO) is therefore considered to be an essential tool to search the large design space, including relevant disciplines simultaneously, and find the global optimum (Bi, 2013). Several MDO approaches have been proposed to address the computational challenges that arise when initiating an MDO process in different industrial applications, for example in the automotive, industrial robot, and aerospace industries (Tarkian, 2012), (Takezawa, 2005), (Amadori, 2012), (Giesing et al., 1998), (Sandberg et al., 2011) and (la Rocca et al., 2011). They consist of, for example, multiple-discipline feasible (MDF) (Kodiyalam et al., 2001), (Balling et al., 1996), All-in-ONE and individual discipline feasible (IDF), (Cramer et al., 1994), Collaborative optimization (Kroo et al. 1994), bio-level integrated synthesis (BLISS) (SobieszczanskiSobieski et al. 1998), concurrent subspace optimization (CSSO), (Sobieszczanski-Sobieski, 1988) and analytical target cascading (Kim et al., 2003). Over the last decade, these methods have been extensively developed to allow MDO to be used in the design process more effectively. But how should the MDO frameworks be applied in a real industrial context using higher fidelity models? This question is either left out or only superficially described. For instance, Hahn (2010) has tried to explain the lack of using higher fidelity geometric model in the conceptual phase and developed a framework called Vehicle Sketch Pad to fill this void. The built-in CAD tool used in this framework may however raise the problem of communicating between this tool and other design tools, as well as technical support for industries to use the framework practically in real applications. CPACS, developed at DLR (The German Aerospace Center, 2013), is designed for use in preliminary airplane design and can successfully handle the complexity of collaborative design in the conceptual phase (Böhnke, 2009).

In most product development processes, the more knowledge gained, the less freedom is left to actually apply the knowledge (Jenkinson et al., 1999) and (Mavris et al., 2000). Fundamental design decisions in the conceptual phase are therefore desirable as these are cheaper than in later phases. However, more accurate knowledge of the product is needed in order to do so successfully. The models currently used at the conceptual level can provide only limited information about the final products. These models therefore need to be replaced with higher fidelity models by using new methods and techniques in modeling and simulation (Kroo et al., 1997). Developing more detailed models is difficult for conceptual engineers who have only superficial knowledge of the final product. As design problems become more complex due to the complexity of the products, system engineering becomes more complicated for conceptual engineers. This leads to greater involvement of domain experts early in the design process. More decentralization of the task, however, may raise the complexity of communication within the design teams. This is especially true in aerospace industries, which require complex analysis in a large design space that involves many disciplines. A straightforward method to control and manage the complexity of system engineering is through Collaborative Design (Kvan, 2000) and (Peng, 1994). In this paper, a collaborative process to set up a design framework in the conceptual phase that includes higher fidelity models is presented. 


\subsection{Collaborative design}

Collaborative design is described by Wang et al. (2002), as " $a$ design process where a product is designed through the collective and joint efforts of many designers". Collaborative design is also referred to as concurrent design or interdisciplinary design (Wang et al., 2002). Designing complex products generally follows a model-driven approach in order to include all related design activities in a collaborative and efficient manner. In the present study, however, the phrase collaborative design is used to describe a more specific kind of collaboration, viz. between conceptual engineers and domain experts.

Manufacturing companies are generally structured into several engineering departments, with domain experts who have specific knowledge about their area of expertise. Domain experts develop high fidelity engineered subsystems. Conceptual engineers, however, are required to define the requirements and overall architecture of a future product.

Domain experts are better suited to develop new subsystems as they tend to have intuitive understanding of the nature of their systems and can better estimate parameters, which are used to predict system and component properties.

Collaboration between engineers AND domain experts can reduce the cycle time in the development of complex systems (La Rocca et al., 2007). This approach brings forth many advantages, some of which are listed below:

- Domain experts can develop models simple enough to be used in a conceptual study and still reflect the performance characteristics of an actual system.

- Verification and validation of models are important tasks. Engineers proficient in their profession are able to conduct verification and validation tasks more effectively, whereas conceptual engineers may not be equipped with the resources to verify the models themselves (Steinkellner, 2011).

- Domain experts have intuitive understanding of the nature of their domains and can better estimate the parameters used to predict system and component properties. They can also estimate technology trends, which can be incorporated into the models (La Rocca et al., 2011).

Nowadays, with a more computerized design process, collaborative design can be made even more efficient. This can be done by developing frameworks which allow simultaneous work on complex systems, reduce manual and sequential operations and ultimately speed up the design process (Wang et al., 2010).

\subsection{Effective computing}

Optimization is not time-efficient, due to the computationally intensive tasks involved. Time has therefore always been one of the drawbacks of using optimization methods in the conceptual phase, where rapid evaluation of the concepts is more important than the accuracy of the result. Surrogate modeling has proven to be an appropriate method to solve this issue by replacing the computationally demanding models in an optimization framework with new, time-efficient models. Surrogate models, or metamodels, are approximate models which are numerically efficient and can mimic the behavior of the system in a given design space (Myers et al., 2009). A surrogate model is created by first generating samples in the design space and performing experiments or simulations of the system at these points. The surrogate model is then fitted to the samples using different methods, e.g. Anisotropic Kriging (Martins et al., 2005). The accuracy of the surrogate model is highly dependent on an efficient sampling and surrogate modeling method. The number of samples, or design of experiments (DOEs), and their placement over the design space therefore has a great impact on accuracy. Uniform Latin Hypercube Sampling (LHS) is used in this work to fit surrogate models 
(Mckay et al., 1979). This method has been used for similar purposes in other researchers' works (Tarkian, 2012) and (Persson, 2012). For more information regarding relevant sampling methods, see (Wang et al., 2002), (Persson, 2012) and (Myers et al., 2009).

The remainder of the paper is structured as follows. Section 2 presents the definitions for Collaborative Multidisciplinary Design Optimization (CMDO). A new tool is proposed to enable a collaborative process in industry. CAVE (Collaborative Aircraft vehicle Engineering), presented in section 3, enables higher fidelity dynamic models to be integrated in conceptual aircraft design. Section 4 explains the solutions developed within the CAVE project by performing a case study where the capability of CAVE within a CMDO framework is presented. Section 5 concludes the paper with a discussion of the results and the challenges that lie ahead as regards assembling an efficient set of tools and processes for future aircraft system design.

\section{Collaborative Multidisciplinary Design Optimization (CMDO)}

Collaborative Multidisciplinary Design Optimization (CMDO) is considered as a new approach to tackling the complexity of multidisciplinary analysis of complex systems in the conceptual design phase. CMDO is a method used to integrate high fidelity design tools in an industrial context. The hierarchical decomposition of the system into subsystems and components enables straightforward integration of the domain experts into the conceptual design. Because the domain experts can develop the model of subsystems based on the new technologies regardless of their effect on the system. For instance, the vapour cycle system as new technology of an aircraft environmental control system (ECS) can be developed and evaluated by the domain experts separately from the ECS.

In the classical approach, the engineering design process starts by conceptual design where the most suited concept to fulfill the requirements is selected by conceptual engineer. The concept is then investigated and optimized more by domain experts using higher fidelity models in later stages of the design process e.g. detail design (Ulrich et al., 2000), (Ullman 1992). The main difference between the proposed CMDO process and classical approach is that in CMDO the experts in detail design phase are involved earlier in order to build a better conceptual models integrate them and optimized them.

The main responsibilities considered in this work include identification of requirements, hierarchical decomposition of subsystems, setting up of interfaces, collaborative tasks, defining design parameters and the sensitivity of objective function regarding each design parameter, and setting up an efficient and less complex optimization aimed at optimizing the system at conceptual level. However Just conceptual engineers are not obviously able to address the above-mentioned responsibilities. The working process can therefore be stated as shown in Figure 1, where four different roles are defined.

- Conceptual engineers (CE): who can decide about the features of a new concept in order to fulfill consumer requirements and preferences. They can also propose suitable approaches to evaluate the concept in terms of quality or performance.

- Domain experts (DE): who are specialists in their domain.

- Interface experts (IE): People with experience from different design departments within the company and who have worked on both higher and lower hierarchy level.

- MDO expert (OE): A person, who is able to formulate the problem, set up the MDO framework, run the optimization, and analyze the results to select the best design parameters based on the requirement(s).

In a large company, the roles outlined in figure 1 may be spread on many persons, whereas at a smaller company developing less complex products it might be possible for one person to have multiple roles. 


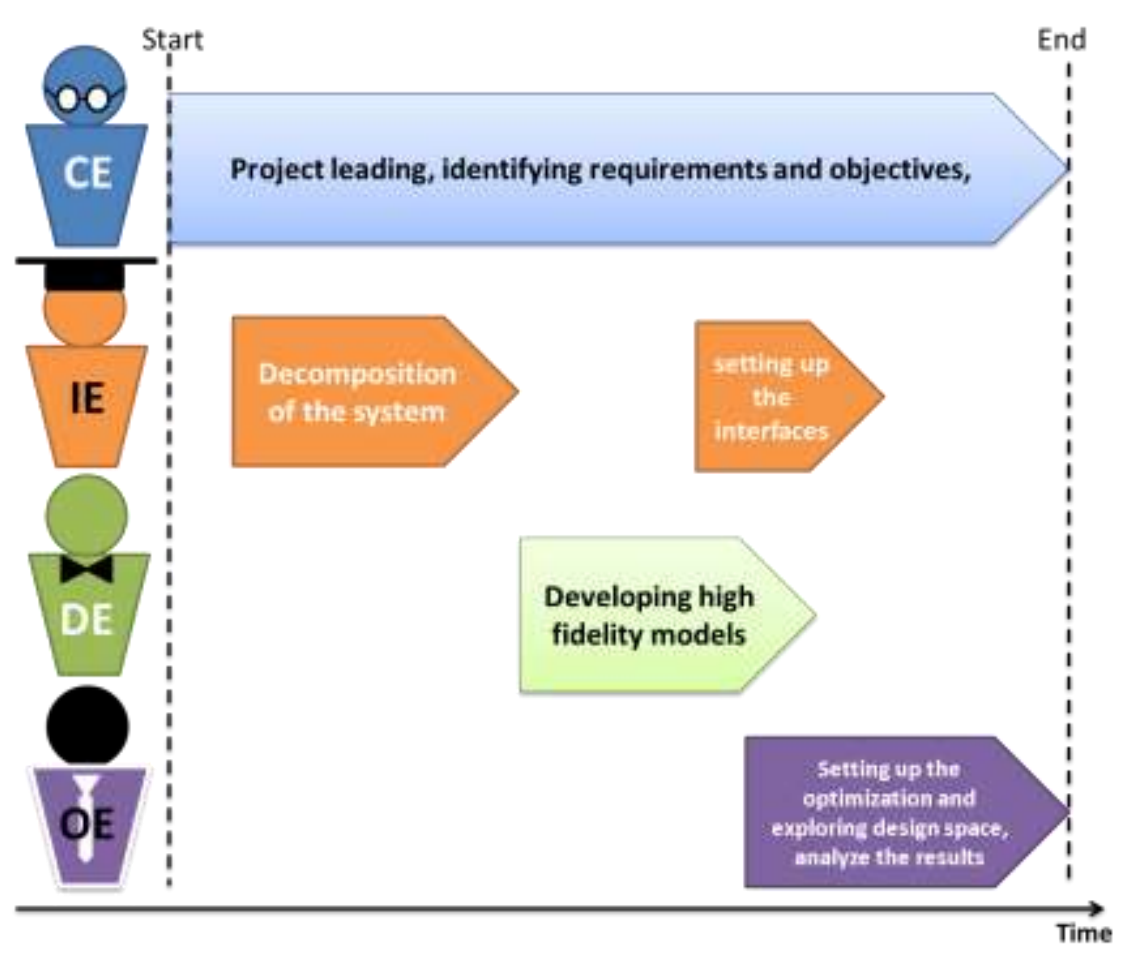

Figure 1: The working process for a CMDO framework

To set up the CMDO framework, the following tasks were performed:

1. Identification of the overall requirements and objectives mainly by CE with the help of the IE.

2. Decomposition of the system which is mainly done by IE.

3. Set up the interfaces in order to fulfill the requirement(s) and provide the objectives mainly done by IE and with consultancy of CE.

4. Development of specific models by DE.

5. Set up an MDO process to optimize the system and analyze the results by ME and CE.

\section{Conceptual aircraft system modeling and optimization}

An aircraft can be viewed as an integrated set of systems - complex with many subsystems and components, multidisciplinary products which are optimized to maintain safe, comfortable and stable flight. The conceptual design of an aircraft vehicle system begins with the definition of requirements and proceeds to a solution at a high abstraction level, see (Wang et Al., 2002).

Up until now, most of the models used at the conceptual level have been empirical and statistical equations based on historical data, which predict optimal design properties such as the power or weight of the system without considering system interactions. The models also have limited possibilities to provide information regarding the size or performance of the systems or their 
components, e.g. volume, hydraulic pressure and voltage of an actuation system. On the other hand, the models are fast and simple, but not easy to develop. (Kroo et al., 1997), (Moir \& Seabridge, 2012), (Raymer, 2011), (Agte et al., 2012).

Geometry and performance are interlinked facets of aircraft design which determine the optimal solution for the vehicle-system architecture, e.g. will the actuator fit in the wing? This information may significantly increase the confidence of the conceptual engineers when selecting one or more suitable architectures. The conceptual models therefore needs to be of higher fidelity to provide more detailed information about the system and facilitate the decision-making process. On the other hand, the detailed models that are typically used in later phases of the design process are complex, slow and not straightforward to deal with (Fritz, 2010), (Steinkellner 2011). Nevertheless, detailed models are much more efficient at providing specific information about the system such as dynamic performance, system interaction, and sizing properties. They are thus not appropriate for conceptual design when effortless, rapid design is an essential requirement.

An industrial CMDO project has been applied and investigated in the research project initiated at Linköping University in collaboration with SAAB Aerospace.

\subsection{Current work process of an aircraft conceptual engineer}

The meaning of conceptual design differs from company to company. Therefore, there would be different approaches in various companies to build and use the conceptual models. Low fidelity models are currently used in the conceptual department in order to provide information about new concepts, mainly on the aircraft level, e.g. power consumption and range. The conceptual engineers design the flight profile based on the customer's requirements and use the models to provide estimates of power consumption, range, and the overall dimensions of the vehicle systems, which are mainly outlined over a vague range. However, they are also very keen to extend their knowledge on system and component levels, e.g. about the size of the aircraft system's components. This can be accomplished using higher fidelity models developed in the modeling and simulation department, which are mainly used for detail analysis. The process in section 2 has been applied in order to use the higher fidelity models early in the conceptual phase. In this study, 3 aircraft subsystems including environmental control system (ECS), Flight control system (FCS) and power generation system, associated with 9 different technologies are considered to be investigated, see Figure 2. High fidelity analysis requires the following models: CAD models, aerodynamic models, and dynamic simulation models. Six domain experts thus need to collaborate in order to create the geometric, aerodynamic, and dynamic models used in CAVE. The domain experts and the MDO expert, From Linköping University who are work together with modeling and simulation department of SAAB, collaborate with an interface expert from SAAB and SAAB's conceptual department as the end user and main customer of the framework.

\subsection{Conceptual aircraft vehicle engineering (CAVE)}

CAVE is made up of aggregations of dynamic models developed in Dymola (Dassault System, 2012) and Excel (Microsoft, 2012) which is the graphical user interface (GUI). In fact, CAVE consists of a set of Modelica models (Fritzson P. 2014) created in Dymola, which represent different technologies that must be evaluated in the conceptual design phase. These models can be controlled and executed through a graphical user interface. For more information regarding the models and working principle of CAVE, please refer to earlier work on CAVE presented in (Safavi et. al. 2012), (Safavi et. al. 2013) and (Safavi, 2013). 


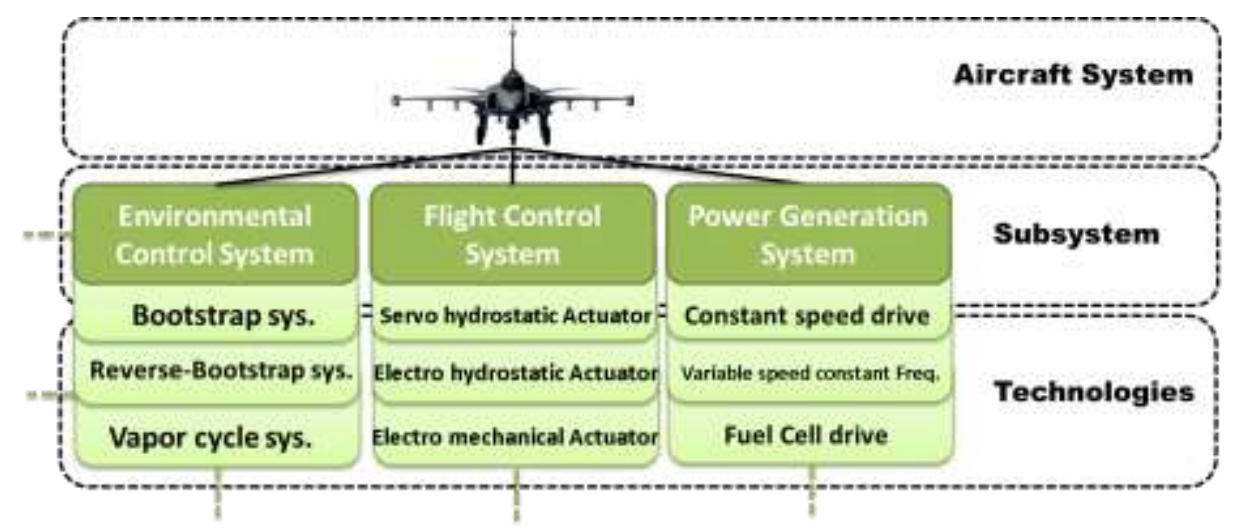

Figure 2: Aircraft, vehicle system, technologies

The system architecture is defined as a set of systems, e.g. an environmental control system and a flight control actuation system. The functionality of any system is associated with one or more technologies, e.g. bootstrap or reverse-bootstrap for an environmental control system (ECS), see Figure 2Figure 2. The technologies consist of different components that can be developed in a collaborative manner with the participation of domain experts.

\subsection{Aircraft Conceptual System Layout}

CAVE is created as an example of a collaborative design tool. The same systematic approach, presented in chapter 2 to create a CMDO framework, is therefore applicable throughout the creation of CAVE. The tasks that have to be completed in order to create a collaborative tool are listed below with some examples from CAVE.

1. Identification of Requirements: These constitute input to the tool on different levels, viz. aircraft level, system level and component level (see Figure 3Figure 3).

- Aircraft level: The basic requirements, e.g. mission profile (altitude, speed, engine thrust, outside temperature, etc., all as a function of mission time) are defined at aircraft level.

- System level: The topology of the system is defined on the system level to determine the main sub-systems and technologies and their connections. For example, in order to simulate the environmental control system (ECS) in an aircraft there is a requirement to connect the ECS to consumers (avionics), heat sinks (fuel tanks or the atmosphere), and energy sources (the main engine, batteries, electrical motors, etc.).

- Component Level: The properties of each technology defined by the working components and their connections. Parameters that determine the basic performance of each component, such as the efficiency, weight and component-specific performance characteristics are defined at component level.

2. Hierarchical decomposition of subsystems: The set of sub-systems that are aggregated to form a complex system can be differentiated according to the sequence of calculation.

- Independent systems - require that the user inputs the load on the system to begin the simulation, e.g. the mission profile. These systems are however not dependent on any other systems. As an example, the actuation system requires the deflection and torque on the flight control surface as input from the user; the system is then simulated in order to calculate, for 
example, the consumed power and the cooling demand, which act as input to the dependent systems.

- Dependent systems - might rely on user input, but depend on the output from other systems in order to begin the simulation. The ECS can be taken as an example where the cooling loads are taken from the simulation of independent and dependent systems.

During a simulation, the independent system is solved first, giving the input to the dependent systems. In some cases, there might also be algebraic loops between dependent systems that need to be resolved using numerical methods.

3. Definition of Interfaces - In this work, the models are designed so that they can be simulated individually; it was therefore found necessary to use a common interface between the systems e.g. power consumed by each system as the interface between systems. However, the interfaces between the components are defined by the characteristics of the system. For instance, the actuation system consumes power during flight and a ratio of the consumed power will generate heat, so the total power consumption will be act as load on the electric system and heat generated will act as load on the cooling system.

\subsubsection{Modeling approach}

When the system layout is set, the systems need to be modeled. The modeling strategy that can help different parties to work more collaboratively is as following:

- The main entity to be analyzed in the main system, e.g. consumed power, is defined as an interface between interacting subsystems. For instance, the FCS and ECS are ran over the predefined flight profile and the power consumed is extracted as an output and then is inputted to the power generation system to provide the required power demanding by FCS and ECS.

- Inverse models - Inverse models can be interpreted such that the meanings of the input and output functions are exchanged. Models developed using the Modelica language are acausal. In an acausal model, a physical system represents by the models consisted by variable and constraints. The variables implicitly expose changes inside models and the relations in a model act as constraints between the variables take at each instant. In a simple word, this means that, there is no distinction between the input and the output of the system. This gives the interface expert more freedom to choose the interfaces based on the objectives.

- The models developed for the conceptual phase can be further improved through inheritance using Modelica's object-oriented features. The models can thus evolve into a higher degree of complexity and be reused in the detailed design stages. 


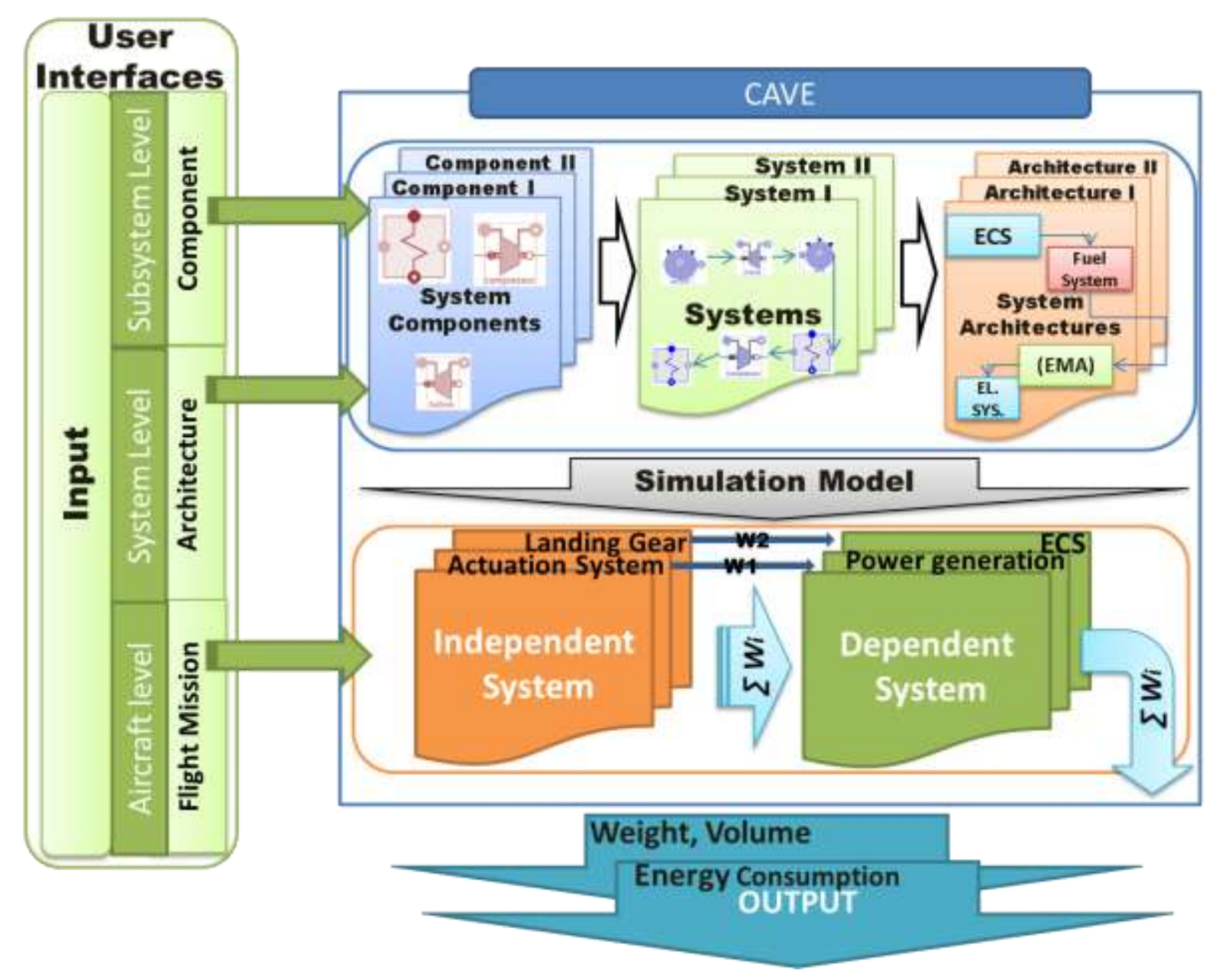

Figure 3: Structure of CAVE and solution sequence in CAVE to calculate the power consumption

\subsubsection{User interface}

The interface is supposed to help the conceptual engineers to focus on model parameter evaluation rather than model development so as to fit the initial requirements. On the other hand, a collaborative tool should be able to bring together all the actors into a single workspace, which in turn increases the effectiveness of the collaboration. The tool should also be able to manage the many complexities that arise from collaboration. Wang et. al. (2002) detailed some situations that can arise, along with a list of tools that provide solutions to the problems. In this project, the collaborative tool chosen was Microsoft Excel because:

- Excel is a widely used tool and most engineers are familiar with it.

- Excel enables collaboration by allowing simultaneous editing of documents when saved in a networked resource. 
- Excel can communicate with other engineering software tools (like Dymola) using Windows COM (Component Object Model) objects which are well documented.

- Specification of requirements can be easily represented using tools like DSM (Design Structure Matrix (Steward, 1981), (Eppinger \& Browning, 2012) in Excel.

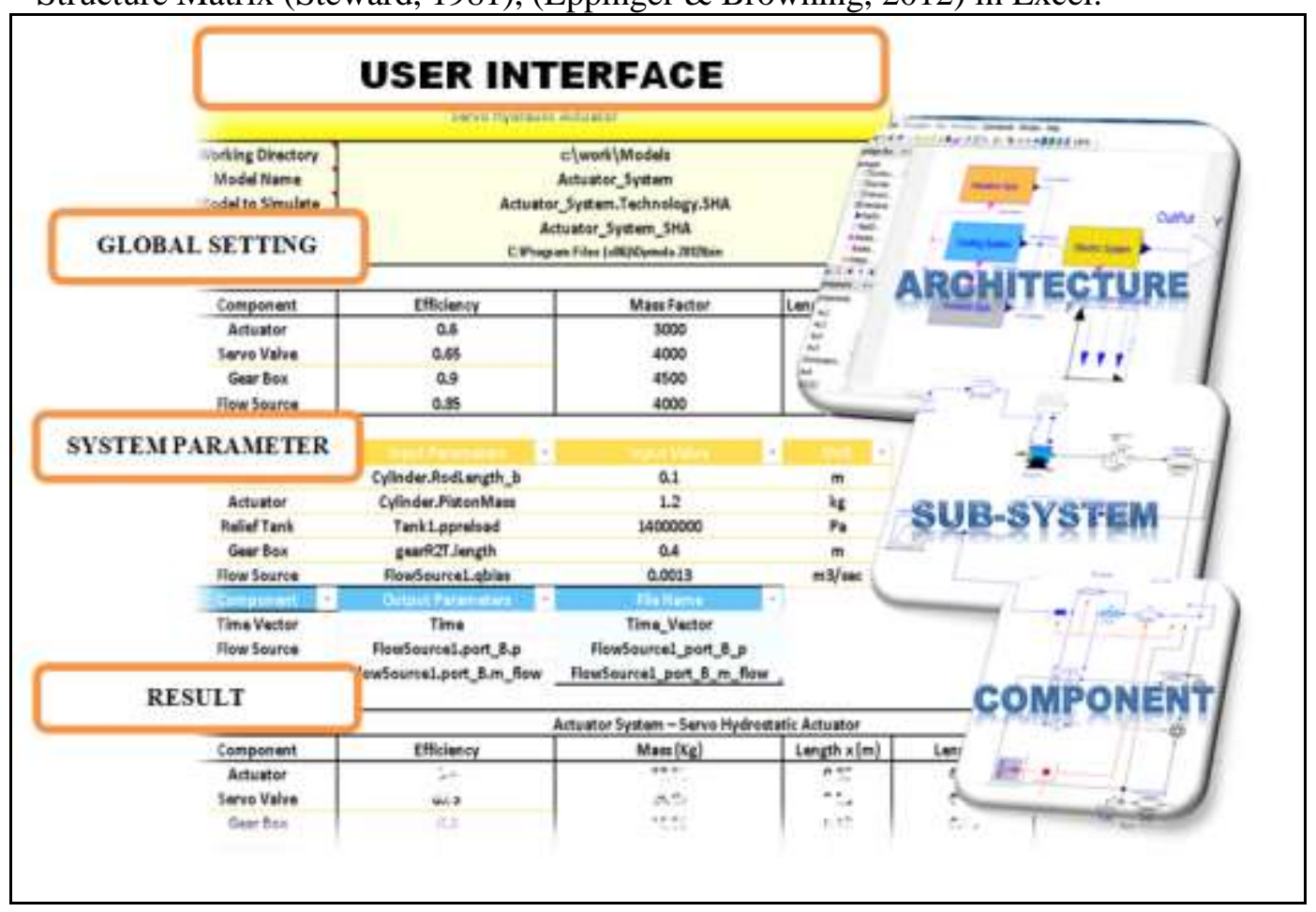

Figure 4: Multiple views of CAVE's user interface

\subsection{System architecture design using CAVE}

During the conceptual phase, many flight system architectures are to be evaluated. Even in a small aircraft, there may be a large number of possible configurations that satisfy the overall requirements. In this section, the collaborative capability of CAVE in evaluation of different system architectures will be presented (See Figure 2Figure 2).

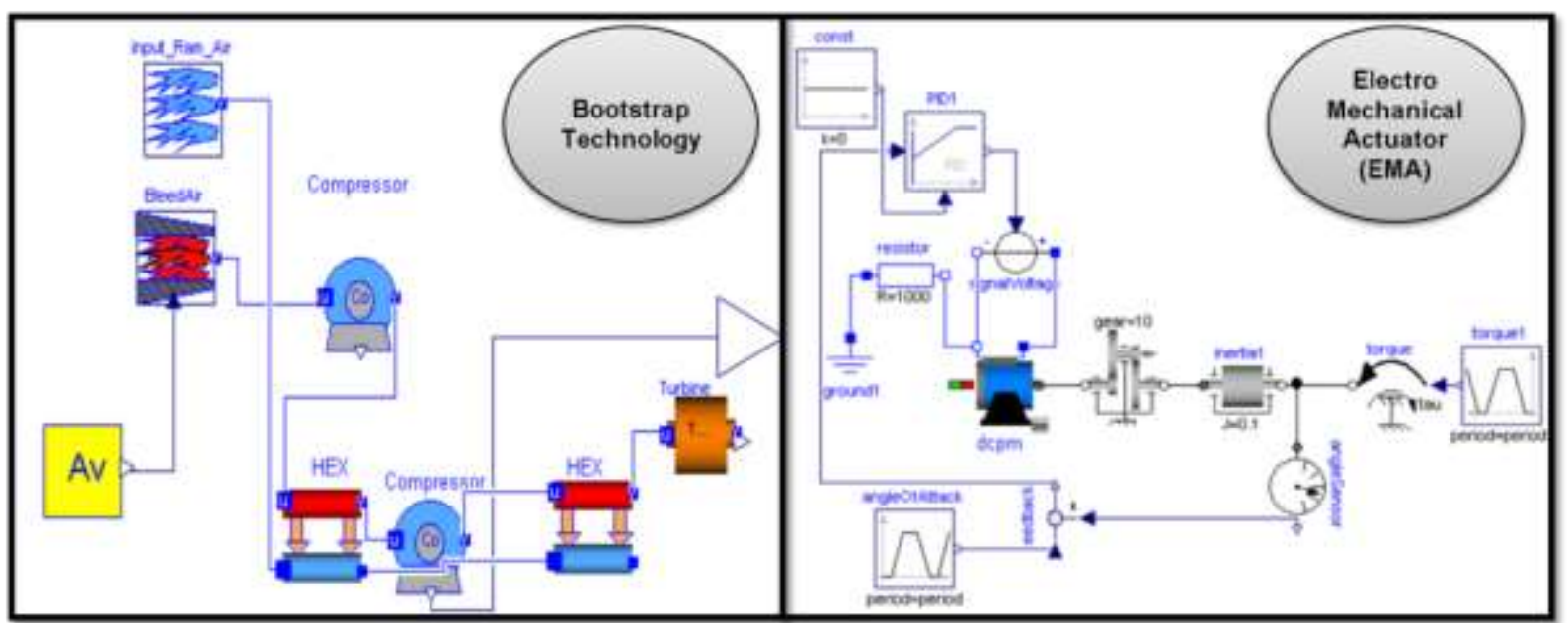

Figure 5: Dymola model of bootstrap technology of ECS system (left) and EMA technology of FCAS (right) 
This study can be used to prove the concept of multidisciplinary design and collaborative design at a conceptual level, which is always a demand in aircraft industries and a difficult task to implement. The entire architecture of the aircraft vehicle system in this study consists of:

- Seven Flight Control Actuation Systems (FCAS) with different technologies such as EMA and Electro hydrostatic actuator (EHA).

- A hybrid cooling system with a bootstrap system and a vapour cycle system with each are responsible to equally provide cooling for heat generating systems.

- One "variable speed constant frequency" power generation system.

The preliminary results show that by using the methods proposed in CAVE, the flexibility of the conceptual engineers to derive empirical data can increase considerably. This can be achieved by evaluating and validating the models based on empirical data, e.g. the component data sheets. For example, Figure 6Figure 6 shows the performance of the cooling system with respect to the cooling that has to be generated. It can be clearly seen that none of the reserved cooling technologies are able to provide the required cooling power. It is also clear that the bootstrap system can reach the necessary capacity only after a certain time. However, this can be improved by changing the design parameters, e.g. increase the ram inlet area or the heat exchangers' parameters. Correspondingly, the maximum power generated by the vapor cycle system is too far from the required power. Hence, using a vapor cycle system seems to be infeasible even with optimized design parameters.

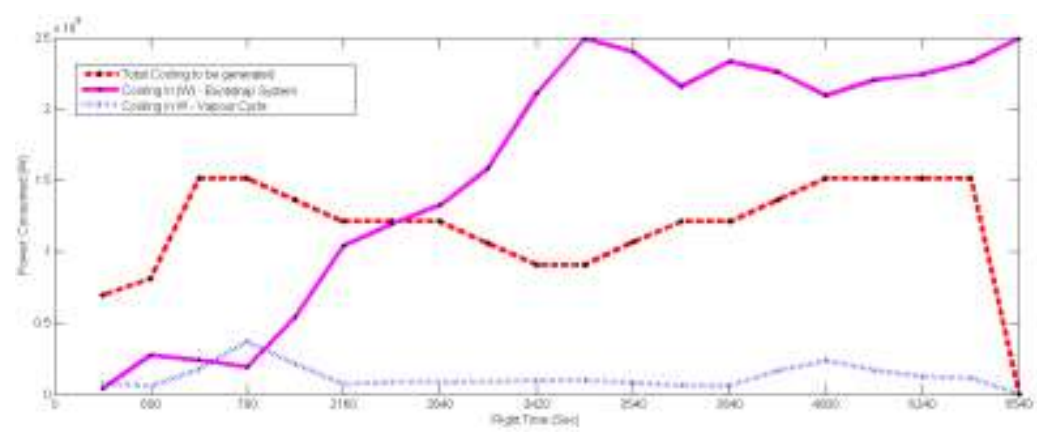

Figure 6: Cooling produced by CAVE's bootstrap and vapor cycle systems

\section{CAVE in a CMDO process}

In order to show how CAVE could be used in a CMDO setting, a case study was performed to optimize the size and power consumed by the actuation system used to control the aircraft flap in the presence of different constraints, e.g. geometrical and aerodynamic constraints. Geometry and aerodynamic performance of an aircraft have direct influence on the performance and efficiency of the aircraft vehicle systems. For instance, dimension and mass properties of an aircraft have a direct effect on the drag which can considerably affect the power consumed by the actuator and consequently the whole aircraft. Calculating the power consumed by the actuation system can therefore be considered to be a multidisciplinary task which needs many parameters to be calculated and exchanged between different models. A collaborative multidisciplinary automated design and evaluation framework is therefore proposed and implemented using modeFRONTIER (modeFrontier 2012), which facilitates the process of integration of various design tools and exchanging information among them. The framework consists of a geometric model (RAPID) (Staack et al., 2012), an aerodynamic model using TORNADO (TORNADO, 2013) and a dynamic model (CAVE), see Figure 7Figure 7.

The working principle behind the framework can be considered to be: 
- The automated geometric tool receives the input from the flight profile, e.g. aircraft overall dimensions, and provides the analysis model with geometric output, e.g. sizes, mass properties, etc. The aerodynamic model requires the size of the aircraft to calculate the aerodynamic forces and torque required to rotate the aircraft flap as well as drag and lift coefficients $(\mathrm{Cd}, \mathrm{Cl})$. The dynamic simulation model of the flight control system (FCS) needs information from the aerodynamic model, e.g. force and torque, to predict the mass and overall dimensions as well as the estimated power consumption of the suggested actuator over a predefined flight profile. If the suggested actuator fits into its position on the wing it is considered to be a feasible solution; otherwise the algorithm iterates to search for another feasible solution, see figure 7 .

- In this framework, computationally expensive simulation models are replaced by surrogate models created in modeFrontier to speed up the optimization. The Anisotropic Kriging method (Martins et al., 2005) was used to create the surrogate model with 300 Uniform Latin Hypercube (ULH) samples. This high number of samples has been chosen to increase the accuracy of the surrogate modeling. To evaluate the model, 50 random samples are generated and used to calculate the error between the original and the surrogate model. The error is calculated using Normalized Root Mean Square Error (NRMSE). A small amount of calculated error $(0.05 \%)$ for the geometric and the aerodynamic model, as well as $1.1 \%$ for the dynamic model, show satisfactory results from the surrogate modeling.

\subsubsection{Optimization formulation}

The main goal of this optimization is to choose suitable actuators from both size and power consumption perspectives that can provide enough torque to rotate the flap.

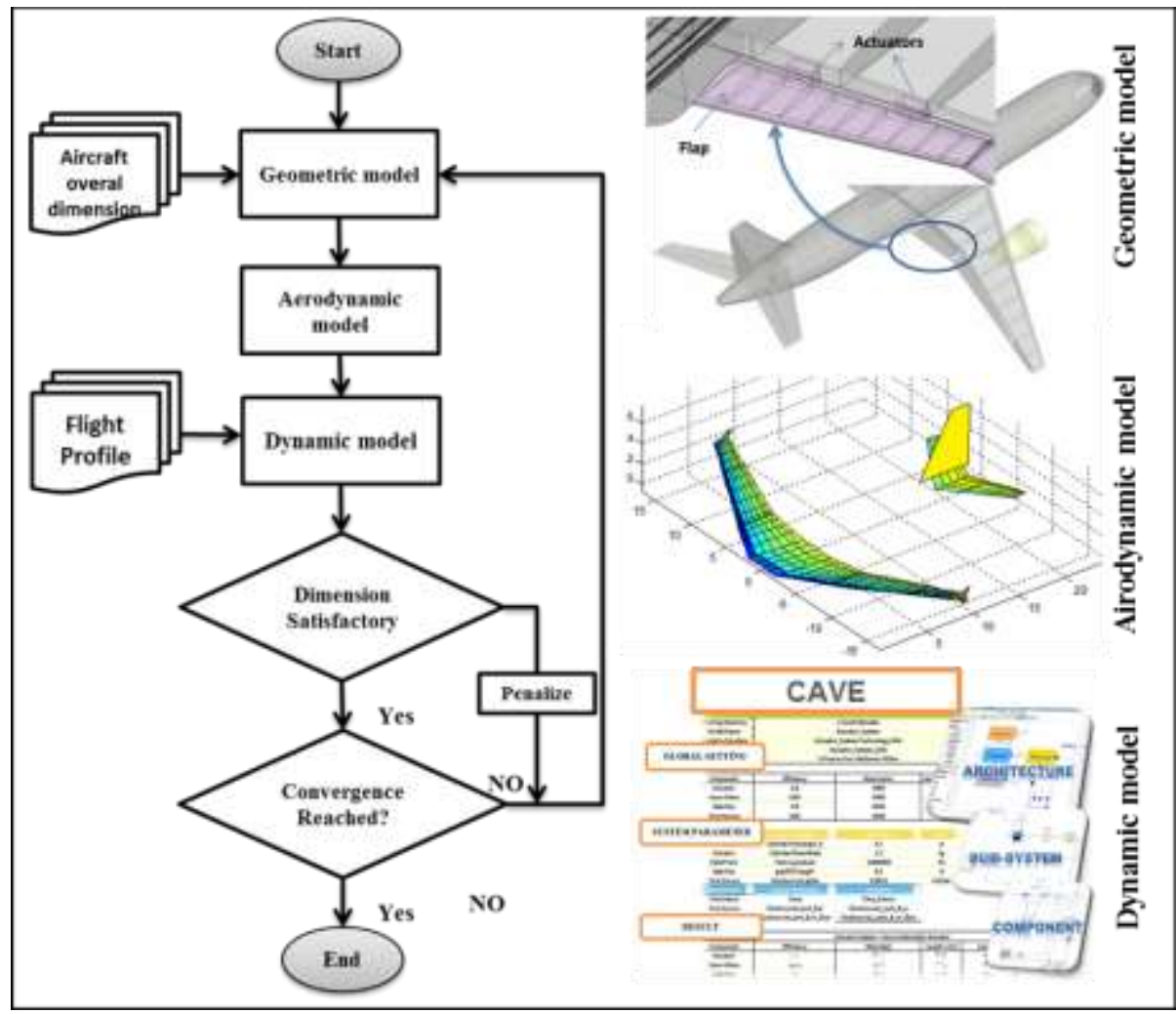

Figure 7: Multidisciplinary Design and Optimization flowcharts (left) and a screen shot of the models involved in the optimization process (right) 
In the problem formulation, the objective function consists of minimizing the power $(P)$ consumed by the actuation system, minimizing the weight of the actuator $(W)$, reducing the weight of the flap $\left(W_{f}\right)$, and the position of the actuator with respect to the fuselage $\left(A_{p}\right)$ due to less force being required to rotate the flap.

The objectives are combined to create the overall objective function of the optimization $(Z)$. Two constraints are defined to ensure that the size of the actuator is always smaller than the corresponding size of its position on the wing. Hence, in the two constraints, geometry $(g 1(x), g 2(x))$ and the volume $\left(A_{v}\right)$ and width of the actuator $\left(A_{\mathrm{w}}\right)$ should be designed smaller than or equal to the volume of the actuator housing $\left(A h_{v}\right)$ and the width of the actuator housing $\left(A h_{w}\right)$ in the aircraft wing, see Eq. (1). The behavior of the actuator can be controlled by the design parameters, such as number of poles in the electric motor $(N)$, current $(I)$, voltage $[V]$, and gear ratio of the gear box $\left(G_{r}\right)$. These also affect the total power and mass of the actuator. Actuator width $(A w)$, actuator position (Ap), and stroke length $(S)$ are other design parameters given as input to the geometric model. The optimization problem can thus be formulated as illustrated in Eq. (1), where $\lambda_{i}$ and $\mu$ are constants that normalize the objective and penalty functions, respectively (Krus et al., 2003).

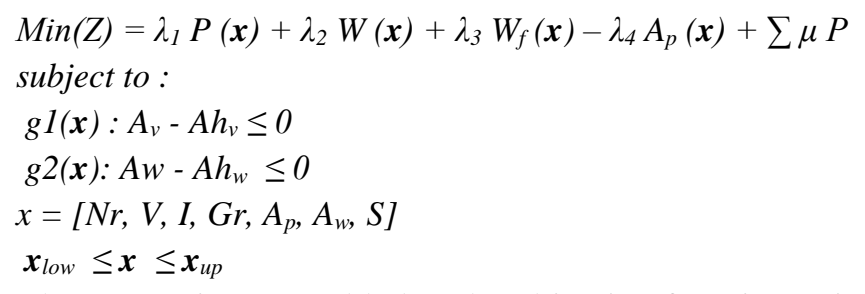

The constraints are added to the objective function using a penalty functions according to Eq. (2):

$P_{1}=\max (0 ; g l(x))^{2}$

$P_{2}=\max (0 ; g 2(x))^{2}$

\subsubsection{Result}

The Simplex algorithm (Nelder-Mead, 1965) is used to solve the optimization problem stated in Eq (1). The algorithm is considered to be a simple optimization algorithm to program and to control and hence a suitable algorithm for the conceptual phase. Optimization time is around 10 minutes on an 8core $3.3 \mathrm{GHz}$ computer. The convergence in the objective function and two of the design parameters, gear ratio and motor current, is shown in Figure 8Figure 8. From an engineering point of view, solving equation 1 is a hard task for conceptual engineers due to the large number of design parameters from various engineering domains. However, the result of this study could be really useful for conceptual analysis because it can give the conceptual engineers an opportunity to evaluate a newly presented technology, e.g. an electric actuator, with respect to the constraints of size and aerodynamic properties of the aircraft. 

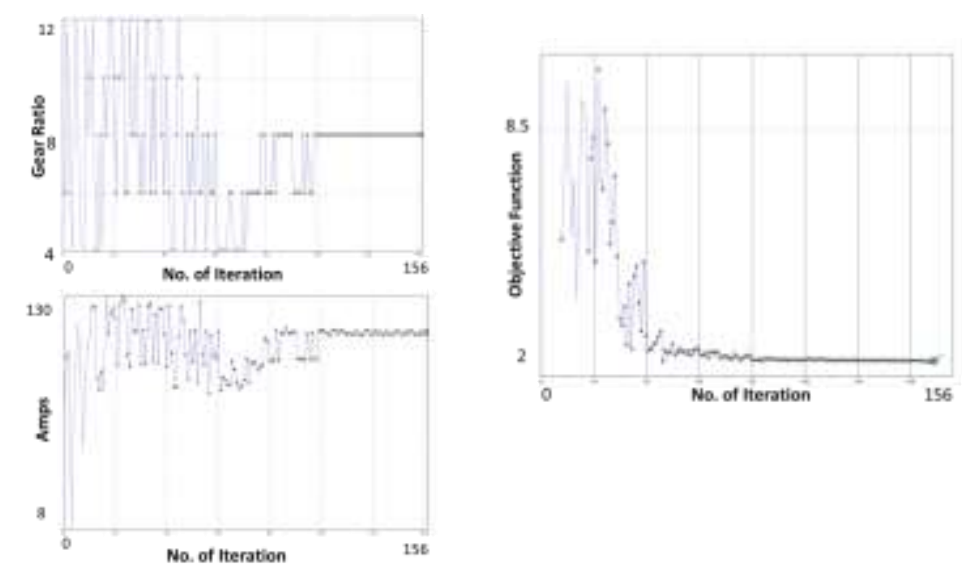

Figure 8: Convergence in objective function (right) and convergence in gear ratio (top left) and current (bottom left)

\section{Discussion and conclusion}

This paper proposes methods to gain more design knowledge early in the conceptual design phase by utilizing a model-based approach where higher fidelity models are brought into a multidisciplinary optimization in the conceptual phase. However, creating higher fidelity models and also managing these models are crucial for conceptual engineers who lack deeper knowledge regarding the entire system and the underlying components. On the other hand, using a model-based approach involves many facets of the design process like requirement analysis, requirement specification, complexity management, model evolution management, model verification, model validation, etc. Highly flexible collaborative design frameworks are thus key enablers to employ more detail models in conceptual design and manage the mentioned complexities. The proposed collaborative multidisciplinary design framework is created through a joint effort by conceptual engineers, domain experts, and MDO and interface experts. The framework enables high fidelity models to be used for concept optimization in the conceptual phase.

Collaborative design (CD) has been proven as a promising method to design the complex product. However, the focus of the researches was mostly on later stages of design and is more dedicated to managing and exchanging information and integrating design tools than to creative team collaboration (Thomas et al, 2012). However, the concentration of this study is mainly on collaboration within the design engineers where a generic procedure to involve the experts in detail design phase into the conceptual phase is presented. The process is evaluated more by doing a case study from the aeronautical industry.

This study presents the potential of using CAVE within a Collaborative MDO process. The results of the optimization also show a satisfactory optimization speed in a conceptual study when using higher fidelity models. In this work process, the conceptual engineers are supposed to work with domain experts, interface experts and MDO experts as illustrated in figure [1]. In the present case study in particular, the conceptual engineer's goal is to design an actuation system to maneuver the aircraft flap for a predefined flight profile based on optimized power consumption and size. The presented results of the framework show the practicability of employing an optimization routine to explore the design space of a complex design problem that requires intricate tools and thus involvement of various experts. 
Since variation in design is high in conceptual design, a substantial number of iterations are required in order to obtain optimized designs. Optimization time thus increases significantly. Surrogate modeling, as proposed in this work, has proven to be a profound method to reduce optimization time. Another benefit of replacing actual models with surrogate models is to reduce the number of occupied software licenses for engineering tools during the optimizations, and hence it is possible to perform optimizations on the concept level without occupying the domain experts' licenses.

\section{References}

Agte J. S., Borer N K., de Weck O., "Multistate Design Approach to Analysis of Twin-Engine Aircraft Performance Robustness" JOURNAL OFAIRCRAFT, Vol. 49, No. 3, May-June 2012

Amadori K. "Geometry Based Design Automation- Applied to Aircraft Design and Optimization" Linköping studies in Science and Technology. Dissertations, 2012.

Balling R. J. and Sobieszczanski-Sobieski J., "Optimization of Coupled Systems: A Critical Overview of Approaches,” AIAA Journal, Vol. 34, No. 1, pp. 6-17, Jan. 1996.

Bi Z., Wang L., Wu C., Yang G., and Zhang D., "Multidisciplinary Design Optimization in Engineering," Mathematical Problems in Engineering, Article ID 351097, 2 pages. doi:10.1155/2013/351097, 2013

Böhnke D., "Data integration in preliminary Airplane Design", Thesis at Distributed Systems and Component Software Department, Institution for Simulation and Software Technology at the German Aerospace Center (DLR), Cologne, 2009.

Brandt S.A., Stiles R.J., Bertin J.J., Whitford R., "Introduction to Aeronautics: A Design Perspective", AIAA Educational Series, Reston, VA, USA, 1997

Breland J. S., Shiratuddin M. F., "A Proposed Framework for Collaborative Design in a Virtual Environment", Advanced Techniques in Computing Sciences and Software Engineering, Springer pp $147-152,2010$

Cheolhan K., Jeongsoo L., Kwangsoo K., Jaeyeol L., Kwangyeol R., “A collaborative design framework for the Korean automotive parts industry" International Journal of Computer Integrated Manufacturing Vol. 26, Iss. 1-2, 2013

Cramer E. J., Dennis J. E., Frank P. D., Lewis R. M. and Shubin G. R., "Problem Formulation for Multidisciplinary Design Optimization," SIAM Journal on Optimization, 4(4), pp. 754-776, November 1994.

Dantzig, G. B. "Linear Programming and Extensions", Princeton University Press, Princeton, NJ, 1963

Eppinger S. D. and Brawning T. R., "Design Structure Matrix Methods and Applications" MIT Press, Cambridge, ISBN: $9780262017527,2012$.

Fritz, K., "System Dependency Analysis Supporting Common Cause Analyses of Complex Aircraft Systems," SAE Int. J. Aerosp. 2(1):28-39, 2010, doi:10.4271/2009-01-3107.

Fritzson P. - "Principles of Object-Oriented Modeling and Simulation with Modelica 3.3: A CyberPhysical Approach", Second edition, John Wiley \& Sons, June 2014.

Giesing JP, Barthelemy, L-FM, “A summary of industry MOD applications and needs", AIAA 19984737, AIAA/USAF/NASA/ISSMO Symposium on Multidisciplinary Analysis and Optimization, St. Louis, MO, USA, Sep.1998.

Hahn, A., "Vehicle Sketch Pad: Parametric Geometry for Conceptual Aircraft Design", 48th AIAA Aerospace Sciences Meeting, Orlando, FL, Jan 4 - 72010. 
Jenkinson L.R., Simpkin P., Rhodes D., "Civil Jet Aircraft Design", Butterworth-Heinemann Publications, Burlington, 1999.

Ko Y.T. "Optimizing product architecture for complex design" Concurrent Engineering 21: 87-102, first published on May 7, 2013 doi:10.1177/1063293X13482472, June 2013.

Kodiyalam S, Sobieszczanski-Sobieski J., "Multidisciplinary design optimization: some formal methods, framework requirements and application to vehicle design". Int. J. Vehicle Design (special issue):3-32, (2001).

Kroo, I.; Altus, S.; Braun, R.; Gage, P.; and Sobieski, I.: " Multidisciplinary Optimization Methods for Aircraft Preliminary Design, " AIAA 94-4325, Fifth AIAA/USAF/NASA/ISSMO Symposium on Multidisciplinary Analysis and Optimization, September 7-9, Panama City, Florida, 1994.

Kvan A., "Collaborative design: what is it? Automation in Construction", Elsevier July 2000.

La Rocca, G., Cerulli, C., Schut, J. E., Nawijn, M., Baluch, H. A., Berends, J., and Tooren, M. J. L. V., "Knowledge Based Engineering supported design: Development of Design and Engineering Engines for multidisciplinary Design and Optimization", Tech. rep., TU Delft. 2011.

La Rocca, G., van Tooren, M.J.L., "Enabling distributed multi-disciplinary design of complex products: a knowledge based engineering approach", J. Design Research, Vol. 5, No. 3, pp.333-352, 2007.

Martins JD. and Simpson TW., "A Method to Manage Uncertainty During System-Level Conceptual Design”, ASE DETC2005-84984, ASME Design Engineering Technical Conference - Design Automation Conference, Long Beach, California, September 24-28, 2005

Mavris D. N. and De Laurentis D. A., "A probabilistic approach for examining aircraft concept feasibility and viability", Aircraft Design, 3 pp.79-101, 2000.

Moir I., Seabridge A., "Design and Development of Aircraft Systems", Volume 67 of Aerospace Series, 2nd edition, John Wiley \& Sons, 2012.

Myers RH., Montgomery DC. and Anderson-Cook CM., "Response Surface Methodology, Process and Product Optimization Using Designed Experiments", Third Version, John Wiley \& sons, Inc., Hoboken, New Jersey, 2009.

Nelder J., Mead R., A Simplex Method for Function Optimization, Computer Journal 7:308-313, 1965.

Pahl G., Beitz, W., Feldhusen, J., Grote, K.-H. "Engineering Design , A Systematic Approach" 3rd ed. Springer 2007.

Peng C., "Exploring communication in collaborative design: co-operative architectural modeling" Journal of Computer-Supported Cooperative Work, 15 (1) (1994), pp. 19-44, 1994

Persson J., "Design and Optimization under Uncertainties, A simulation and Surrogate Model Based Approach" Licentiate thesis, Linkoping University, 2012

Raymer D. P., "Conceptual Design Modeling in the RDS-Professional Aircraft Design Software" AIAA Aerospace Sciences Meeting, Orlando, FL, 2011

Safavi E., Chaitanya M.V.R.., Ölvander J., Krus P., "Multidisciplinary Optimization of Aircraft Actuation System for Conceptual Analysis“, AIAA 51st Aerospace Sciences Meeting, Grapevine, Texas, 2013.

Safavi E., Gopinath V., Ölvander J., Gavel H., "A Collaborative Tool for Conceptual Aircraft Systems Design" AIAA Modeling and Simulation Technologies, Minneapolis, Minnesota, 2012.

Sandberg M., Tyapin I., Kokkolaras M., Isakasson O., Aidanpää J.-O. and Larsson T. “A Knowledgebased Master-model Approach with Application to Rotating Machinery Design" Concurrent Engineering December 2011 19: 295-305, first published on October 17, 2011 
Sobieszczanski-Sobieski J. "Optimization by decomposition: a step from hierarchic to nonhierarchicsystems". NASA Technical Report CP-3031, 1988.

Sobieszczanski-Sobieski J., Agte J. and Sandusky R., "Bi-Level Integrated System Synthesis (BLISS)," Proceedings,7th AIAA/USAF/NASA/iSSMO Symposium on Multidisciplinary Analysis and Optimization, AIAA, St. Louis, Missouri, September 1998.

Steinkellner S., "Aircraft Vehicle Systems Modeling and Simulation under Uncertainty", Licentiate thesis, Linkoping University, 2011.

Steward, D.V. "Systems Analysis and Management: Structure, Strategy and Design", New York: Petrocelli Books, ISBN 0-894-33106-X, 1981.

Takezawa A., Nishiwaki S., Izui K., Yoshimura M., Nishigaki H., and Tsurumi Y. "Concurrent Design and Evaluation Based on Structural Optimization using Structural and Function-oriented Elements at the Conceptual Design Phase" Concurrent Engineering March 2005 13: 29-42,

Tarkian M. "Design Automation for Multidisciplinary Optimization- A High Level CAD Template Approach" Linköping studies in Science and Technology. Dissertations, 1479- 2012.

Thomas E., Ravachol M., Quincy J.B. and Malmheden M., "Collaborative complex system design applied to an aircraft system", Proceedings of the 9th International Modelica Conference 855 10.3384/ecp12076855, Munich, Germany September 3-5, 2012.

Ullman D., "The Mechanical Design Process", McGraw-Hill Inc. New York, 1992.

Ulrich, K.T., Eppinger, S.D., “ Product Design and Development $2^{\text {nd }}$ Edition”, Irwin McGraw-Hill, Boston, 2000.

Wang Q., Zhong-Wei R., Zhong-Feng G., "XML-based data processing in network supported collaborative design". International Journal of Automation and Computing August 2010, Volume 7, Issue 3, pp 330-335. 2010.

Wang, L. H., Shen, W. M., Xie, H., Neelamkavil, J., and Pardasani, A., "Collaborative conceptual design - state of the art and future trends", COMPUTER AIDED DESIGN, Vol. 34, No. 13, 2002, pp. 981996- 2002.

Wodehouse, A. J., \& Ion, W. J. "Information use in conceptual design: Existing taxonomies and new approaches". International Journal of Design, 4(3), 53-65, 2010.

World Wide Web Consortium, Extensible Markup Language (XML), http://www.w3.org/XML/ 2012 